\title{
Decreased ZONAB expression promotes excessive transdifferentiation of alveolar epithelial cells in hyperoxia-induced bronchopulmonary dysplasia
}

\author{
ANA HOU, JIANHUA FU, YONGYAN SHI, LIN QIAO, JUN LI, YUJIAO XING and XINDONG XUE \\ Department of Pediatrics, Shengjing Hospital of China Medical University, Shenyang, Liaoning 110004, P.R. China
}

Received March 7, 2017; Accepted January 10, 2018

DOI: $10.3892 / \mathrm{ijmm} .2018 .3413$

\begin{abstract}
Previous studies by our group have confirmed excessive transdifferentiation of alveolar epithelial cells (AECs) in a hyperoxia-induced bronchopulmonary dysplasia (BPD) model, but the underlying mechanism have remained elusive. The transcription factor zonula occludens 1-associated nucleic acid binding protein (ZONAB) has the biological functions of inhibition of epithelial cell differentiation and promotion of epithelial cell proliferation. The aim of the present study was to explore the regulatory effect of ZONAB on the transdifferentiation and proliferation of AECs in a model of hyperoxia-induced lung injury. Newborn Wistar rats were randomly allocated to a model group (inhalation of $85 \% \mathrm{O}_{2}$ ) or a control group (inhalation of normal air), and ZONAB expression in lung tissues was detected at different time-points. Type II AECs (AEC II) isolated from normal newborn rats were primarily cultured under an atmosphere of 85 or $21 \% \mathrm{O}_{2}$, and ZONAB expression in the cells was examined. The primary cells were further transfected with ZONAB plasmid or small interfering (si)RNA and then exposed to hyperoxia, and the indicators for transdifferentiation and proliferation were measured. The present study indicated that ZONAB expression in AEC II of the BPD rats was significantly decreased from 7 days of exposure to hyperoxia onwards. In the AEC II isolated from normal neonatal rats, ZONAB expression in the model group was also reduced compared with that in the control group. After transfection with the plasmid pCMV6-ZONAB, the expression of aquaporin 5 (type I alveolar epithelial cell marker) decreased and the expression of surfactant protein C (AEC II marker), proliferating-cell nuclear antigen and cyclin D1 increased, which was opposite to the effects of ZONAB siRNA. Transfection with pCMV6-ZONAB also alleviated
\end{abstract}

Correspondence to: Professor Xindong Xue, Department of Pediatrics, Shengjing Hospital of China Medical University, 36 Sanhao Street, Shenyang, Liaoning 110004, P.R. China E-mail: xdxue1111@126.com

Key words: ZO-1-associated nucleic acid binding proteins, alveolar epithelial cell, transdifferentiation, proliferation, hyperoxia, bronchopulmonary dysplasia excessive transdifferentiation and inhibited proliferation of AEC II induced by hyperoxia treatment. These results suggest that ZONAB expression in AEC II decreases under hyperoxia conditions, which promotes transdifferentiation and inhibits proliferation of AECs. This may, at least in part, be the underlying mechanism of lung epithelial injury in the hyperoxia-induced BPD model.

\section{Introduction}

With the increasing level of neonatal treatment, an increasing proportion of premature children born at an early gestational age and with a low birth weight survive, although the subsequent incidence of preterm birth-associated complications continues to increase. Bronchopulmonary dysplasia (BPD) is a chronic respiratory disease, which is one of the most common sequelae of premature birth, particularly in those $<30$ weeks gestational age $(1,2)$. According to the Neonatal Research Network of the National Institute of Child Health and Human Development, the incidence of the new classification of severity-based BPD has been reported to be as high as $68 \%$ among preterm infants born between gestational weeks 22 and 28 (traditional definition, 42\%; physiological definition, 40\%) (3). BPD not only causes relatively high infant mortality soon after birth, but can also induces multiple respiratory and nervous system complications during adolescence $(1,4,5)$.

Alveolar simplification and disorganized vasculature are the major pathological characteristics of 'new' BPD $(6,7)$. It is widely accepted that neonatal hyperoxia promotes a BPD-like pathology characterized by alveolar simplification and angiogenic impairment $(8,9)$. Simplified alveolar epithelium in the lungs was alsoobserved in previous studies on hyperoxia-treated neonatal rats by our group $(10,11)$. A compensatory increase in transdifferentiation of alveolar epithelial cells (AECs) was identified in the lungs of the hyperoxic group rather than their suppression (10). In addition, decreased proliferation of type II AECs (AEC II) was observed in vitro, which may, at least partially, contribute to the development of simplified alveolar epithelium in hyperoxia (10). However, the factors affecting such changes of AECs have remained elusive.

Zonula occludens 1 (ZO-1)-associated nucleic acid-binding protein (ZONAB) is a Y-box transcription factor that is recruited to tight junctions (TJs) by binding to the $\mathrm{Src}$ homology 3 domain of ZO-1 $(12,13)$ and has selectivity for 
sequences of specific promoters containing an inverted CCAAT box (12). ZONAB shuttles between the TJs and nuclei, while the sequestration with $\mathrm{ZO}-1$ at the TJs prevents accumulation of ZONAB in the nucleus, where ZONAB engages in transcriptional activities (13-15). As a transcription factor, the first discovered role of ZONAB was the promotion of cell proliferation. Cyclin-dependent kinase (CDK) 4 is a core factor that regulates $\mathrm{G} 1 / \mathrm{S}$ transition in the cell cycle, with transposition between the nucleus and cytoplasm (16). Balda et al (13) reported that ZONAB modulates the accumulation of CDK4 in the nucleus to affect the regulation of the cell cycle. When the concentration of ZONAB in the nucleus is reduced, CDK4 is reduced and cell proliferation is subsequently inhibited. Proliferating-cell nuclear antigen (PCNA) is a basic regulator of eukaryotic DNA replication and repair (17). Sourisseau et al (18) confirmed that PCNA is the target gene of ZONAB and that PCNA expression is upregulated by increased ZONAB expression, which promotes DNA replication and cell proliferation. Cyclin D1, the core factor of cell cycle regulation, is also a target gene of ZONAB (18-20). Cyclin D1 expression is upregulated by overexpression of ZONAB, which activates CDK4 and CDK6 in order to promote G1/S transition (18). At the same time, ZONAB also inhibits epithelial cell differentiation. During normal kidney development, epithelial cells undergo a transition from proliferation to differentiation, resulting in polarization of the epithelial cell layers. However, this polarization disappears during tumor formation, resulting in excessive cell proliferation and regression of tissue repair. Expression of the megalin and cubilin genes is a marker of epithelial differentiation (21). Lima et al (22) identified the megalin and cubilin genes as target genes of ZONAB, and ZONAB was able to decrease megalin and cubilin expression and inhibit epithelial cell differentiation. ZONAB expression in human renal clear cell carcinoma tissue is abnormally increased, resulting in further proliferation of differentiated cells, loss of normal polarized morphology and promotion of tumorigenesis.

Therefore, the present study focused on ZONAB expression in AECs in a hyperoxia-induced BPD model and its possible function in the regulation of the transdifferentiation and proliferation of AECs. The primary objectives of the present study were to determine whether the transcription factor ZONAB was differentially expressed in the lung of newborn rats exposed to hyperoxia and to identify an alternative pathway that promotes BPD-like alveolar simplification.

\section{Materials and methods}

Animal model. All animal procedures were approved by the Medical Ethics Committee of Shengjing Hospital affiliated to China Medical University (Shenyang, China). BPD animal models were established according to methods previously described by our group (10). Adult Wistar rats (age, 3-4 months old; 20 females of weight 250-300 g and 5 males of weight 350-400 g) were purchased from the Animal Lab of the Experimental Research Center of Shengjing Hospital (China Medical University, Shenyang, China) and mated at a male to female ratio of 1:4. The 211 newborn Wistar rats were bred in house and randomly divided into two groups: A BPD model group, which inhaled $85 \%$ oxygen and a control group, which inhaled air. The rats were housed in the following conditions: 12-h light/dark cycle; temperature, $25-26^{\circ} \mathrm{C}$; humidity, $60-70 \%$; boxes were opened for $30 \mathrm{~min}$ each day to add water and feed, and replace bedding. Food and water were available ad libitum. The oxygen concentration in the glass chamber was monitored with an oxygen measurement instrument (model no. 572; Servomex, Inc., Norwood, MA, USA). Maternal rats in the two groups were switched every $24 \mathrm{~h}$ to avoid oxygen toxicity. Neonatal rats were kept under constant hyperoxia $(8,10)$ and were not kept for longer than 21 days.

Lung sample collection. According to a method previously described by our group (10), eight rats were randomly selected from the two groups on days 1,3,7, 14 and 21, and under intraperitoneal anesthesia (chloral hydrate $300 \mathrm{mg} / \mathrm{kg}$ body weight), the chest cavity was immediately opened and the left atrial appendage was cut. Subsequently, the right ventricle was punctured with a sterile infusion needle and irrigated with saline solution at a pressure of $18 \mathrm{~cm} \mathrm{H}_{2} \mathrm{O}$ to wash out any blood in the lungs. The left lung tissue was fixed in $4 \%$ paraformaldehyde, embedded in paraffin and routinely sliced into 4- $\mu \mathrm{m}$ tissue sections, which were stained with hematoxylin (5 min) and eosin (1-3 min) at room temperature. For the preparation of frozen sections, the tissues fixed in $4 \%$ paraformaldehyde were dehydrated in $30 \%$ sucrose for $12 \mathrm{~h}$ and frozen at $-80^{\circ} \mathrm{C}$. Subsequently, the tissues were sliced into $7-\mu \mathrm{m}$ sections for immunofluorescence double staining. The right lung tissue was placed in Eppendorf tubes and frozen at $-80^{\circ} \mathrm{C}$ for western blot and reverse transcription-quantitative polymerase chain reaction (RT-qPCR) analyses.

AEC II isolation and culture. In accordance with the method described in a previous study by our group (10), 200 newborn rats within $24 \mathrm{~h}$ after birth were anesthetized and lavaged to isolate cardiopulmonary tissues, which were subsequently placed in pre-chilled D-Hanks solution. The isolated lung tissue was repeatedly washed to remove residual blood, cut into small pieces $\left(1 \mathrm{~mm}^{3}\right)$ and repeatedly rinsed in D-Hanks solution. The pieces of tissue were immersed in $0.25 \%$ Trypsin-EDTA solution (Gibco; Thermo Fisher Scientific, Inc., Waltham, MA, USA) in each well and incubated for $30 \mathrm{~min}$ at $37^{\circ} \mathrm{C}$ in a water bath. The digestion was terminated by the addition of an equal volume of $10 \%$ fetal bovine serum (FBS; Gibco; Thermo Fisher Scientific, Inc.). The mixture was filtered through a 200 -mesh filter and then centrifuged $\left(4^{\circ} \mathrm{C}, 235 \mathrm{x} \mathrm{g}, 5 \mathrm{~min}\right)$. The supernatant was removed and the pellet was incubated with $0.1 \%$ collagenase I (Gibco; Thermo Fisher Scientific, Inc.) for $20 \mathrm{~min}$ at $37^{\circ} \mathrm{C}$ in a water bath. The mixture was centrifuged again $\left(4^{\circ} \mathrm{C}, 235 \mathrm{x} \mathrm{g}, 5 \mathrm{~min}\right)$ and the pellet was resuspended in medium containing $10 \% \mathrm{FBS}$, then inoculated in a Petri dish and incubated in a humidified atmosphere of $5 \% \mathrm{CO}_{2}$ at $37^{\circ} \mathrm{C}$. The medium was replaced three times at $50-\mathrm{min}$ intervals to remove adherent fibroblasts. Non-adherent cells were transferred to rat immunoglobulin $\mathrm{G}$ ( $\operatorname{IgG}$ )-coated dishes for $1 \mathrm{~h}$ to remove macrophages. The cells were adjusted to a density of $2-3 \times 10^{6}$ cells $/ \mathrm{ml}$ and inoculated in a $50-\mathrm{ml}$ flask or six-well plate to continue the culture. After $24 \mathrm{~h}$, relatively pure AECs II were obtained by removing blood cells and dead cells. Of the cells, $>95 \%$ were viable as demonstrated by trypan blue staining, with $90 \%$ purity by immunofluorescence 
staining against surfactant protein C (SP-C). After $24 \mathrm{~h}$ of culture, the cells were randomly allocated to a model group or a control group. Cells in the model group were incubated in an atmosphere of $85 \% \mathrm{O}_{2} / 5 \% \mathrm{CO}_{2}$ in a standard incubator (CB150; Binder GmbH, Tuttlingen, Germany) and the control group was maintained under an atmosphere of $21 \% \mathrm{O}_{2} / 5 \% \mathrm{CO}_{2}$ in a Series 113111 Water Jacketed $\mathrm{CO}_{2}$ Incubator (Thermo Fisher Scientific, Inc.) for $48 \mathrm{~h}$ of continuous culture.

The extraction of AEC II from the rats in the BPD model group and control group was performed on days 7, 14 and 21 after hyperoxia or air exposure, in accordance with specific methods for extraction, as described above. The cells were purified by differential adhesion and $\operatorname{IgG}$ adhesion, centrifuged, and the supernatant was discarded. The cells were stored at $-80^{\circ} \mathrm{C}$ for western blot and RT-qPCR analyses.

Trypan blue staining. Dead cells can be stained by Trypan blue, but live cells prevent the dye from entering; therefore, trypan blue staining may be used to distinguish between dead and live cells. A single-cell suspension $\left(10^{6}\right.$ cells $\left./ \mathrm{ml}\right)$ was prepared and mixed with $0.4 \%$ trypan blue solution. Cells were loaded into a hemocytometer and observed under inverted phase contrast microscope. The cell viability was determined as the total number of live cells divided by the sum of the total number of live and dead cells, multiplied by 100 .

Immunofluorescence staining. The primary and secondary antibodies for double staining of ZONAB/SP-C in lung tissue were polyclonal rabbit anti-ZONAB (1:50 dilution; cat. no. 402800; Invitrogen; Thermo Fisher Scientific, Inc.), DyLight 488 AffiniPure donkey anti-rabbit IgG (H\&L; 1:100 dilution; cat. no. E032221-02; EarthOx Life Sciences, San Francisco, CA, USA), polyclonal goat anti-SP-C (1:100 dilution; cat. no. sc-7705; Santa Cruz Biotechnology, Inc., Dallas, TX, USA) and donkey anti-goat IgG (H\&L) Affinity Pure, DyLight594 Conjugate (1:100 dilution; cat. no. DkxGt-003-D; ImmunoReagents, Raleigh, NC, USA). The cultured cells were incubated with polyclonal rabbit anti-ZONAB (1:50 dilution; cat. no. 402800; Invitrogen; Thermo Fisher Scientific, Inc.) and DyLight 549 AffiniPure goat anti-rabbit IgG (H\&L; 1:100 dilution; cat. no. E032320; EarthOx Life Sciences) for ZONAB staining. The primary and secondary antibodies for immunofluorescence double staining of cultured cells for aquaporin (AQP)5/SP-C were polyclonal rabbit anti-SP-C (1:50 dilution; cat. no. sc-13979; Santa Cruz Biotechnology, Inc.), DyLight 488 AffiniPure donkey anti-rabbit IgG (H\&L; 1:100 dilution; cat. no. E032221; EarthOx Life Sciences), polyclonal goat anti-AQP5 (1:100 dilution; cat. no. sc-9891; Santa Cruz Biotechnology, Inc.) and donkey anti-goat IgG (H\&L) Affinity Pure, DyLight594 Conjugate (1:100 dilution; cat. no. DkxGt-003-D; ImmunoReagents). Immunofluorescence staining of lung tissue was performed with frozen sections (7 $\mu \mathrm{m}$ ) fixed in methanol for $30 \mathrm{~min}$ at room temperature and blocked with $10 \%$ FBS for 30 min at room temperature, which were then washed and incubated with primary antibody at room temperature overnight, washed and then incubated with immunofluorescence secondary antibody at room temperature for 3-4 h, washed, and incubated for $5 \mathrm{~min}$ with DAPI (1:1,000 dilution; Sigma-Aldrich; Merck KGaA, Darmstadt, Germany). Immunofluorescence was then observed under a confocal laser-scanning microscope (C1; Nikon Corp., Tokyo, Japan). Immunofluorescence staining of cultured AECs was performed on tissue culture-treated polycarbonate filter inserts (Transwell; Corning-Costar, Corning, NY, USA), which were fixed with $4 \%$ paraformaldehyde for $30 \mathrm{~min}$ at room temperature and then fixed in methanol for another $30 \mathrm{~min}$ at room temperature, followed by incubation with primary antibody overnight at $4^{\circ} \mathrm{C}$. The remaining steps were identical to those for immunofluorescent staining of tissue.

Western blot analysis. Total protein was extracted from lung tissues or cells using lysis buffer for western blot analysis (cat. no. P0013; Beyotime Institute of Biotechnology, Shanghai, China). EpiQuik Nuclear Extraction kit (EpiGentek Group, Inc., New York, NY, USA) was used to extract total nuclear protein from cells. Protein concentration was determined by a Bradford assay, and equal amounts of protein $(50 \mu \mathrm{g})$ from extracts were mixed with sample loading buffer. After electrophoresis on $12 \%$ precast SDS-PAGE gels (70 V for $90 \mathrm{~min}$ ), proteins were transferred to polyvinylidene difluoride membranes (cat. no. LC2005; Thermo Fisher Scientific, Inc.) (70 $\mathrm{V}$ for $90 \mathrm{~min})$. The membranes were blocked with $5 \%(\mathrm{w} / \mathrm{v})$ nonfat milk in a buffer of $100 \mathrm{mM}$ Tris base $(\mathrm{pH} 7.5)$ containing $0.9 \%(\mathrm{w} / \mathrm{v}) \mathrm{NaCl}$ and $0.1 \%(\mathrm{v} / \mathrm{v})$ Tween $20(\mathrm{TBST})$ for $1 \mathrm{~h}$ at room temperature and then incubated overnight at $4^{\circ} \mathrm{C}$ with the following primary antibodies: Rabbit anti-ZONAB (1:800 dilution; Invitrogen; Thermo Fisher Scientific, Inc.), goat anti-AQP5 (1:500 dilution; Santa Cruz Biotechnology), rabbit anti-SP-C (1:500 dilution; Santa Cruz Biotechnology), mouse anti-PCNA (1:1,000 dilution; Abcam, Cambridge, MA, USA), rabbit anti-cyclin D1 (1:1,000 dilution; Abcam), rabbit anti-LAMIN B1 (cat. no. ab16048; 1:1,000 dilution; Abcam) or rabbit anti- $\beta$-actin (1:1,000 dilution; Sigma-Aldrich; Merck KGaA). Following extensive washing in TBS-T three times, the membranes were incubated with horseradish peroxidase-conjugated secondary antibody (Cell Signaling Technologies, Inc., Beverly, MA, USA) at room temperature for $2 \mathrm{~h}$. The membranes were then washed and treated with western blotting luminol reagent (cat. no. sc-2048; Santa Cruz Biotechnology, Inc.) and the signal was visualized using an Alpha Ease RFC Imaging System (Alpha Innotech Corp., San Leandro, CA, USA). Integrated density values of the protein bands were analyzed using a Fluor Chen 2.0 system (Bio-Rad Laboratories, Inc., Hercules, CA, USA) and normalized to those of $\beta$-actin or LAMIN B.

$R T-q P C R$. Total RNA was extracted from lung tissues and cells using TRIzol reagent (Invitrogen; Thermo Fisher Scientific, Inc.). A total of $1 \mu \mathrm{g}$ RNA was reverse-transcribed into complementary DNA using the SuperScript III kit (Invitrogen; Thermo Fisher Scientific, Inc.) according to the manufacturer's instructions. IQ SYBR Green Supermix (Bio-Rad Laboratories, Inc.) and a 7500 Real-Time PCR System (Applied Biosystems; Thermo Fisher Scientific, Inc.) were used for quantitative real-time PCR. Gene-specific primers were designed and synthesized by Takara Biotechnology (Dalian, China). The primer sequences are listed in Table I. The threshold cycle was recorded to reflect the mRNA expression levels and dissociation curve analysis was performed to ensure the specificity of the PCR products. The relative expression of 
Table I. Primer sequences.

\begin{tabular}{lll}
\hline Gene name & \multicolumn{1}{c}{ Forward primer $\left(5^{\prime} \rightarrow 3^{\prime}\right)$} & Reverse primer $\left(5^{\prime} \rightarrow 3^{\prime}\right)$ \\
\hline ZONAB & CCCTACAACTATAGGCGCCGTTC & GCACTGCTCTGTTCGGTAGCTG \\
AQP5 & CTCCGAGCTGTCTTCTACGTG & CAGGCGTTGTGTTGTTGTTC \\
SP-C & GGTAGCAAAGAGGTACTGATGG & CACCACGACGACAAGGACTA \\
PCNA & TAAGGGCTGAAGATAATGCTGAT & CCTGTTCTGGGATTCCAAGTT \\
$\beta$-actin & ACCGTGAAAAGATGACCCAGAT & CAGTGGTACGACCAGAGGCATA
\end{tabular}

ZONAB, zonula occludens 1-associated nucleic acid binding protein; SP-C, surfactant protein C; PCNA, proliferating cell nuclear antigen; AQP, aquaporin.

target gene/internal reference mRNA was expressed according to the $2^{-\Delta \Delta \mathrm{Cq}}$ method (23).

Transfection of ZONAB plasmids. AEC II was isolated from neonatal rat lungs and cultured in six-well cell plates at a density of $2-3 \times 10^{6}$ cells $/ \mathrm{ml}$. The cells were incubated at $37^{\circ} \mathrm{C}$ in an atmosphere of $5 \% \mathrm{CO}_{2}$ for $24 \mathrm{~h}$. The plasmid DNA clones for rat ZONAB and the empty vector pCMV6 were purchased from Origene Technologies, Inc. (Rockville, MD, USA) and were amplified using an endotoxin-free plasmid Maxi-prep kit (Qiagen, Valencia, CA, USA). The cells were transfected at a ratio of $1 \mu \mathrm{g}$ plasmid DNA to $2 \mu \mathrm{l}$ Lipofectamine 2000 (Invitrogen; Thermo Fisher Scientific,Inc.) in $100 \mu 1$ Opti-MEM Reduced Serum Media (Invitrogen; Thermo Fisher Scientific, Inc.), according to the manufacturer's protocol. After incubation at room temperature for $20 \mathrm{~min}$, the transfection complex was added to the cell culture. Cells were cultured for $6 \mathrm{~h}$, and the medium with the transfection solution was then removed and replaced with complete Dulbecco's modified Eagle's medium: Nutrient mixture F-12 (DMEM/F12) (Gibco; Thermo Fisher Scientific, Inc.). The cells were harvested after $48 \mathrm{~h}$ and subjected to the abovementioned assays. The AEC viability assay was performed using trypan blue staining.

Transfection of small interfering RNA (siRNA) of ZONAB. Cultured AEC II were seeded into six-well plates at a density resulting in $70 \%$ confluence. The siRNA sequence targeting rat ZONAB (siZONAB; 5'-CCGAAACGACAC CAAAGAAdTdT-3') was designed and synthesized by Guangzhou RiboBio Co., Ltd. (Guangzhou, China). siZONAB or control non-targeting siRNA complexed with $2 \mu 1$ Lipofectamine 2000 in $100 \mu \mathrm{l}$ of Opti-MEM Reduced Serum Media was added concurrently and incubated at room temperature for $20 \mathrm{~min}$. The transfection complex was then gently added to the cells. The medium was replaced with complete DMEM/F12 medium after $6 \mathrm{~h}$. Cells were analyzed after $48 \mathrm{~h}$. The cell viability was determined by trypan blue staining.

Statistical analysis. All analyses were performed using SPSS 18.0 software (SPSS, Inc., Chicago, IL, USA). Values are expressed as the mean \pm standard deviation and the mean values were obtained from at least three independent experiments. Statistical significance between two groups was determined using Student's t-test. One-way analysis of variance followed by Dunnett's test was used for multiple group comparisons. $\mathrm{P}<0.05$ was considered to indicate a statistically significant difference.

\section{Results}

Animal condition. The mortality rate as a result of hyperoxia was $\sim 6.7 \%$, and the mortality rate observed in the present study as a result of hyperoxia was expected according to our previous studies (data not shown). The signs of hyperoxia-induced toxicity were poor response, reduced activity, dull body hair, low body weight, short body height, high mortality, as well as cyanosis and shortness of breath following oxygen exposure, which were in agreement with previous studies $(10,11,17)$. These symptoms were considered to be humane endpoints in the present study. In the present study, $1 \%$ of the animals exhibited a body weight loss $>20 \%$ and were immediately sacrificed. Although infrequent, this phenomenon has been observed in previous studies $(10,11,17)$. Animals meeting the humane endpoints were subjected to cardiac puncture following intraperitoneal anesthesia with chloral hydrate (300 mg/kg body weight). Animal death was confirmed by lack of heartbeat.

$Z O N A B$ expression is deregulated in the lung tissues of the $B P D$ model group. ZONAB expression in lung tissues of the model group and the control group was first detected and the total amount of ZONAB protein expressed in the lung tissues was determined by western blot analysis. The results demonstrated that, as compared with the control group, ZONAB expression increased from days 1-21 of hyperoxia (Fig. 1A). In order to eliminate interference with other cellular components in lung tissues and to specifically observe ZONAB expression in AEC II, the lung tissue sections were assessed using immunofluorescence double staining. As presented in Fig. 1B, the AEC II-specific marker SP-C was labeled with red fluorescence, ZONAB was labeled with green fluorescence, and double-stained cells (yellow) represent AEC II expressing ZONAB. The results indicated that ZONAB expression in the AEC II of the model group was significantly reduced compared with that in the control group (Fig. 1B). Conversely, excessive expression of ZONAB was identified in the bronchial epithelial cells of the model group (Fig. 1C). At the same time, AEC II was isolated and purified from the lungs of the control and model groups at different time-points (days 7, 14 and 21), and the target protein and mRNA were extracted from 
A

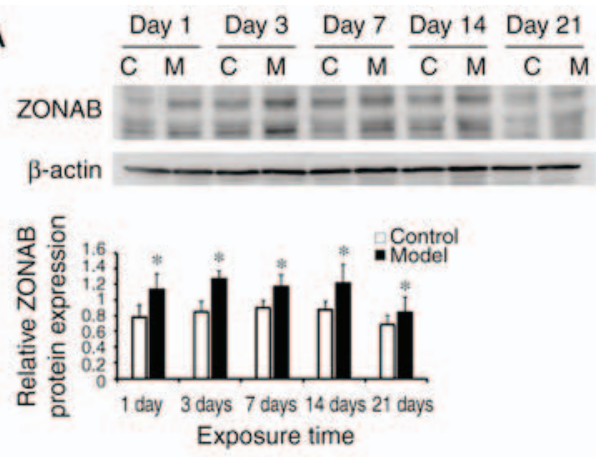

D

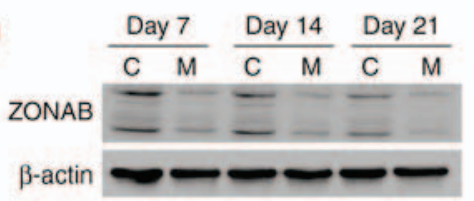

B

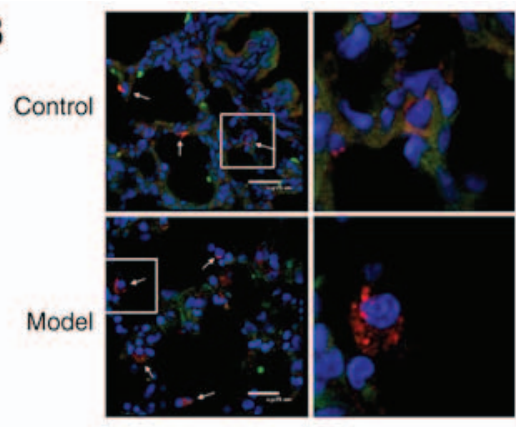

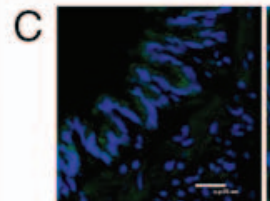

Control

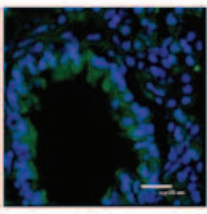

Model
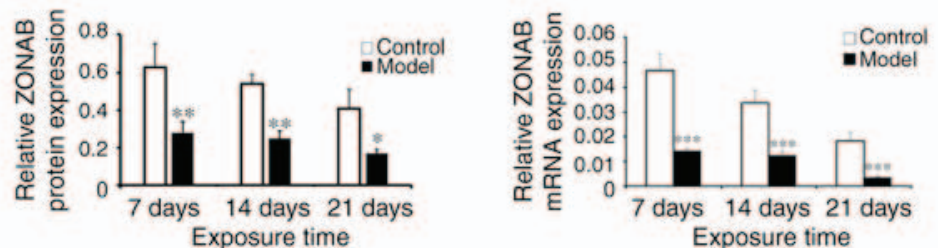

Figure 1. Abnormal ZONAB expression in lung tissues of the bronchopulmonary dysplasia model group. (A) Western blot analysis was used to measure ZONAB expression in lung tissues of the control and model groups. (B) At day 21, lungs of animals from the two groups were double-stained (magnification, x800; scale bar, $25 \mu \mathrm{m}$ ). SP-C, the AEC II marker, is labeled with red fluorescence and ZONAB is labeled with green fluorescence. Double-stained cells are displayed in yellow, representing AEC II expression of ZONAB. ZONAB expression in the AEC II was significantly decreased in the model group (shown by arrows). Images in the boxes were magnified and displayed in the right-hand panel to indicate ZONAB and SP-C localization (magnification, x800; scale bar, $25 \mu \mathrm{m}$ ). (C) Immunofluorescence staining of lung tissues on day 21 demonstrated excessive expression of ZONAB in bronchial epithelial cells in the model group (magnification, x800; scale bar, $25 \mu \mathrm{m}$ ). (D) Western blot analysis was used to measure the protein expression, while reverse-transcription quantitative polymerase chain reaction analysis was employed to analyze the mRNA expression of ZONAB in AEC II isolated from lung tissues of rats in the control and model groups. ${ }^{*} \mathrm{P}<0.05,{ }^{* *} \mathrm{P}<0.01,{ }^{* * *} \mathrm{P}<0.001$ vs. control group on the same day. $\mathrm{C}$, control group; $\mathrm{M}$, model group; ZONAB, zonula occludens 1 -associated nucleic acid binding protein; SP-C, surfactant protein C; d, days; AEC II, type II alveolar epithelial cells.

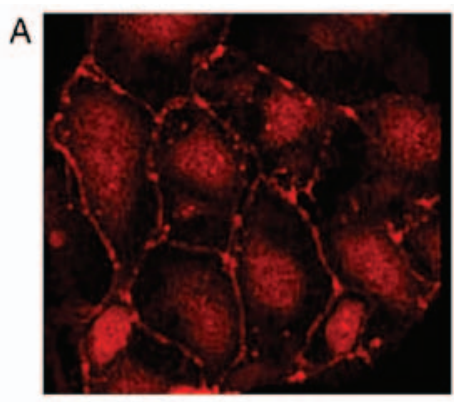

Control

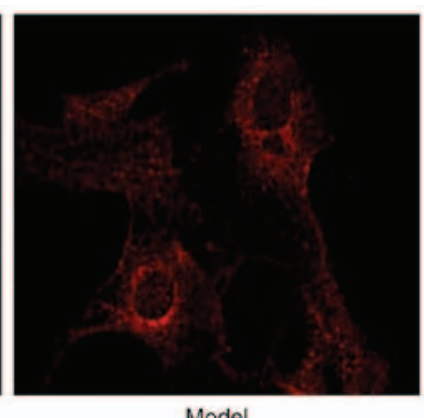

Model
B

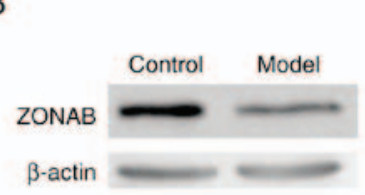

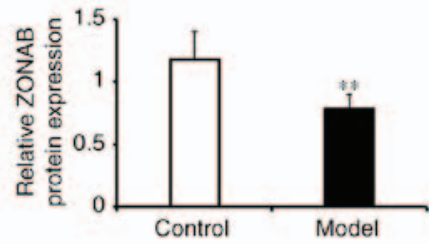

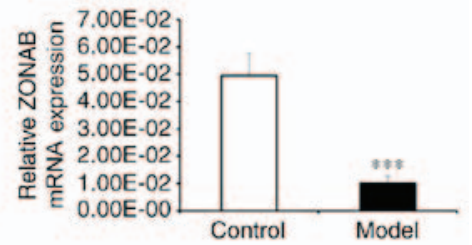

Figure 2. In vitro ZONAB expression in primary cultured AEC II. (A) ZONAB immunofluorescence staining of primary cultured AEC II (magnification, $\mathrm{x} 800$ ). ZONAB is labeled by red fluorescence. (B) Western blot analysis was used to determine the protein expression, while reverse-transcription quantitative polymerase chain reaction analysis was employed to analyze the mRNA expression of ZONAB in primary cultured AEC II. ${ }^{* *} \mathrm{P}<0.01,{ }^{* * *} \mathrm{P}<0.001$ vs. control group. ZONAB, zonula occludens 1-associated nucleic acid binding protein; AEC II, type II alveolar epithelial cells.

the obtained cell samples for further quantification (Fig. 1D). The results indicated that ZONAB expression in the control and model groups gradually decreased with prolonged exposure time, while the average ZONAB expression in the model group was lower than that in the control group $(\mathrm{P}<0.05)$.

ZONAB expression in primary cultured AEC II. In order to better understand the effect of ZONAB on the transdifferentiation and proliferation of AECs, an in vitro AEC II model was established. The expression levels and cellular localization of ZONAB were detected by immunofluorescence staining, which demonstrated that compared with that in the model group, the expression of ZONAB in the control group was higher, and that the protein was mainly located at the cellular junctions or in the nuclei, whereas in the model group, it was aggregated in the cytoplasm with little remaining at the cell junctions or in the nuclei (Fig. 2A). Western blot and RT-qPCR analyses were used to quantify the expression 

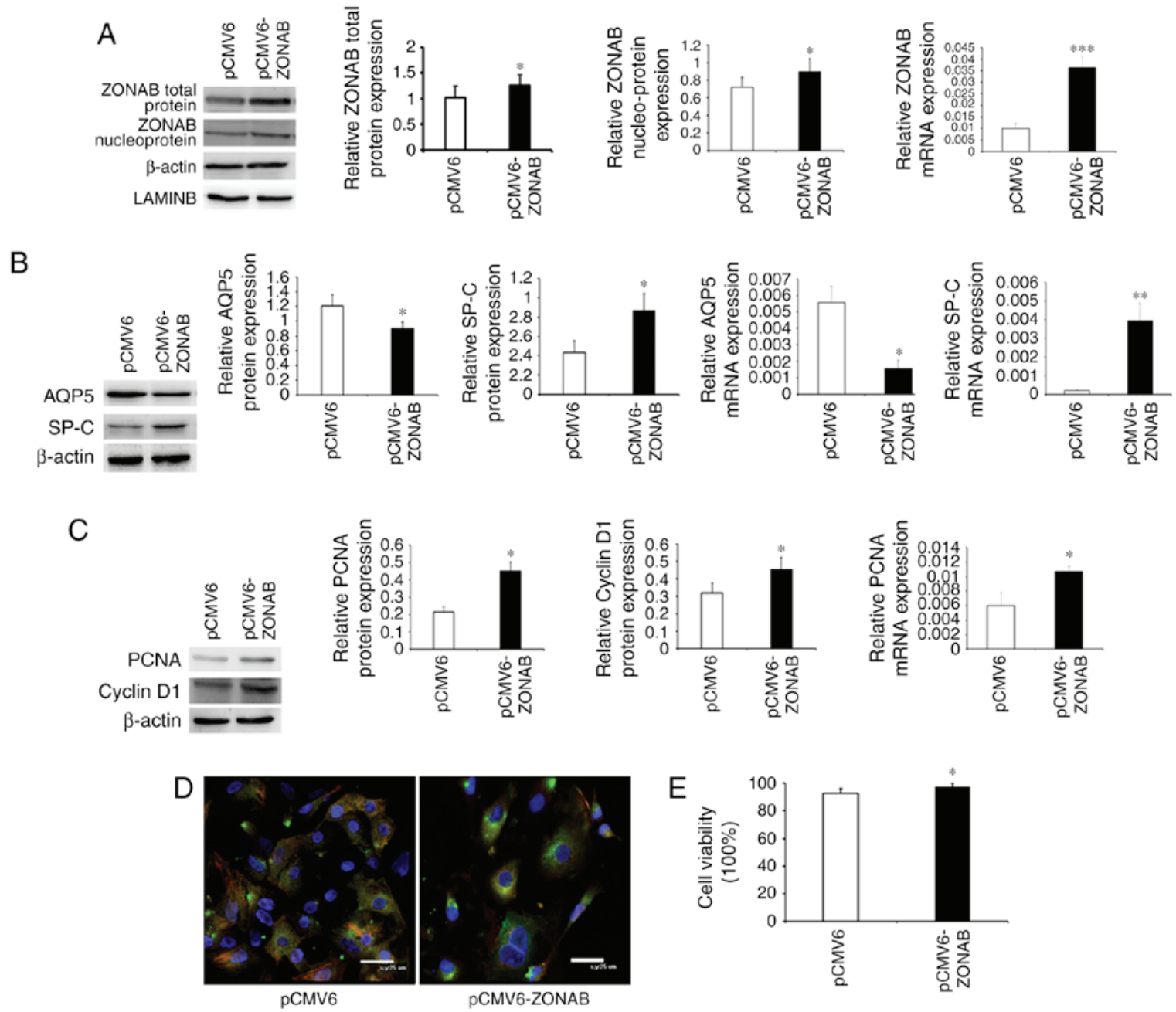

Figure 3. Overexpression of ZONAB inhibits transdifferentiation and promotes proliferation of AEC II. (A) Expression of ZONAB protein and mRNA in the pCMV6-ZONAB-transfected and blank control groups. (B) Protein and mRNA expression of AQP5 and SP-C in the two groups of cells. (C) Protein and mRNA expression of PCNA and cyclin D1 in the two groups. (D) Immunofluorescent double staining results for the two groups of cells (magnification, x800; scale bar, $25 \mu \mathrm{m}$ ). Red fluorescent-labeled AQP5 represents AEC I, green fluorescent-labeled SP-C represents AEC II, and the double-stained cells are displayed in yellow, representing cells transformed from AEC II to AEC I. The nuclei were stained with DAPI (blue). (E) Cell viability in the two groups. ${ }^{*} \mathrm{P}<0.05,{ }^{* *} \mathrm{P}<0.01,{ }^{* * * *} \mathrm{P}<0.001$ vs. pCMV6 group. ZONAB, zonula occludens 1 -associated nucleic acid binding protein; SP-C, surfactant protein C; AEC II, type II alveolar epithelial cells; PCNA, proliferating cell nuclear antigen; AQP, aquaporin; pCMV, cytomegalovirus plasmid.

of ZONAB protein and mRNA, both of which suggested that ZONAB expression in the model group was lower than that in the control group (Fig. 2B).

ZONAB overexpression inhibits transdifferentiation and promotes proliferation of AEC II. ZONAB expression was decreased in AEC II of the BPD model group and a previous study by our group had confirmed increased transdifferentiation and decreased proliferation of AEC II in the BPD model group (10). In the present study, it was further verified whether ZONAB is involved in the regulation of transdifferentiation and proliferation of AEC II. ZONAB expression in primary AEC II was detected by RT-qPCR and western blot analyses after pCMV6-ZONAB transfection. The results indicated that $\mathrm{ZONAB}$ protein and $\mathrm{mRNA}$ levels in the pCMV6-ZONAB-transfected group were significantly higher than those in the blank control group $(\mathrm{P}<0.05)$. ZONAB protein expression in the nuclei was also significantly increased compared with the level of the LAMIN B control (Fig. 3A) (24). The expression of the AEC I marker AQP5 and the AEC II marker SP-C was examined, which indicated that AQP5 expression in the pCMV6-ZONAB-transfected group was significantly lower than that in the control group, and SP-C expression was significantly increased, suggesting decreased transdifferentiation (Fig. 3B). The expression levels of PCNA and cyclin D1 in the pCMV6-ZONAB-transfected group were significantly higher than those in the control group (Fig. 3C). Cell immunofluorescence double staining demonstrated that AQP5 expression was decreased, while SP-C expression was increased in the pCMV6-ZONAB-transfected group. The double-stained cells in the pCMV6-ZONAB-transfected group were reduced compared with those in the control group (Fig. 3D), which indicated decreased transdifferentiation and increased proliferation. The cell viability assay performed by trypan blue staining indicated increased cell viability in the pCMV6-ZONAB-transfected group (Fig. 3E).

Knockdown of ZONAB expression promotes transdifferentiation and inhibits proliferation of AEC II. In order to further investigate the effects of ZONAB on the transdifferentiation 

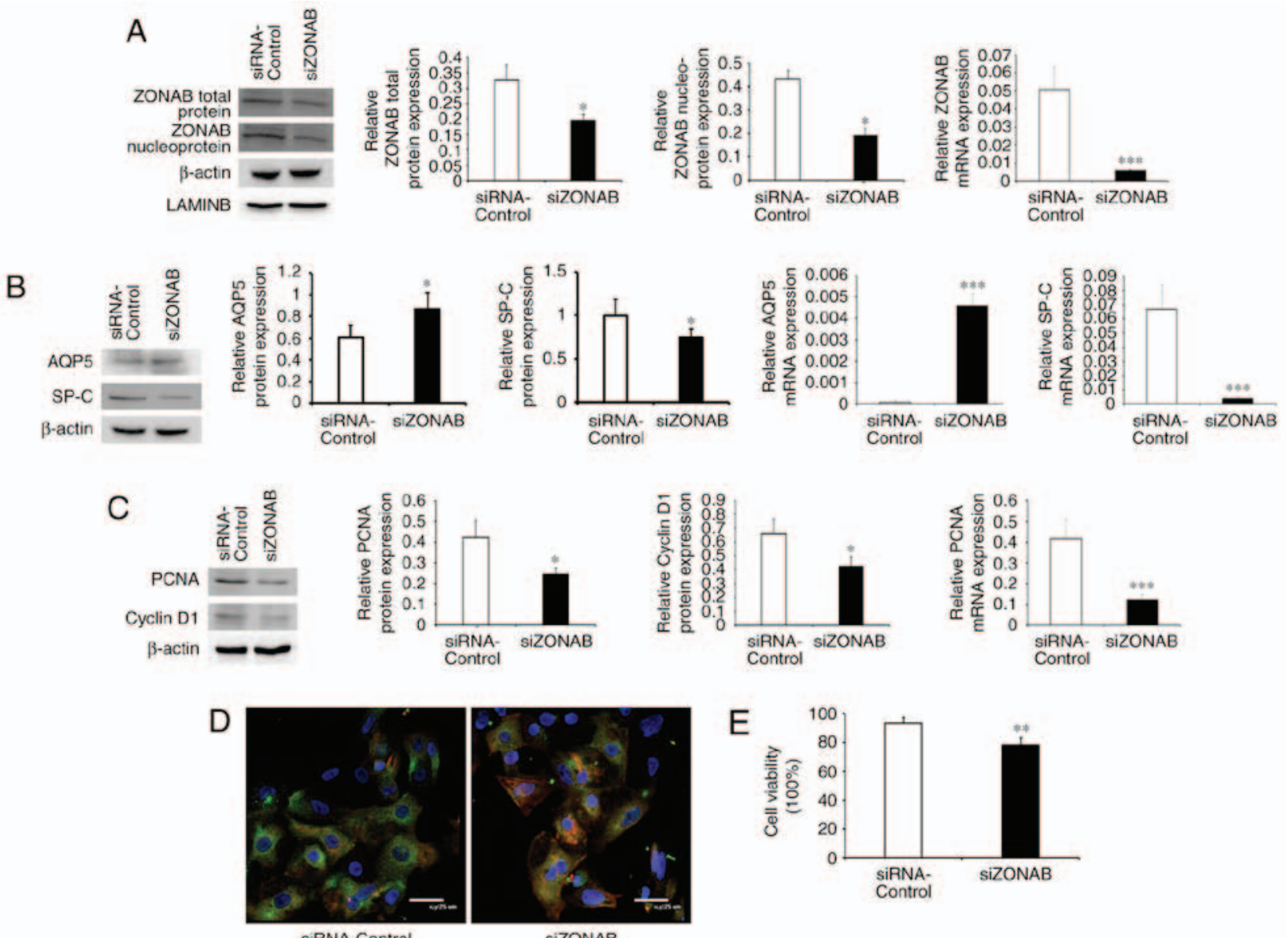

Figure 4. Knockdown of ZONAB expression promotes transdifferentiation and inhibits proliferation of AEC II. (A) Protein and mRNA expression of ZONAB in AEC II of the siZONAB-transfected and blank control groups. (B) Protein and mRNA expression levels of AQP5 and SP-C in the two groups of cells. (C) Protein and mRNA expression of PCNA and cyclin D1 in the two groups. (D) Immunofluorescent double staining results for the two groups of cells (magnification, x800; scale bar, $25 \mu \mathrm{m}$ ). The red fluorescent-labeled AQP5 represents AEC I, the green fluorescent-labeled SP-C represents AEC II, the double-stained cells (yellow) represent cells transformed from AEC II to AEC I, and nuclei were stained with DAPI (blue). (E) Cell viability in the two groups. ${ }^{*} \mathrm{P}<0.05,{ }^{* *} \mathrm{P}<0.01,{ }^{* * *} \mathrm{P}<0.001$ vs. control group. ZONAB, zonula occludens 1 -associated nucleic acid binding protein; SP-C, surfactant protein C; AEC II, type II alveolar epithelial cells; PCNA, proliferating cell nuclear antigen; AQP, aquaporin; siRNA, small interfering RNA.

and proliferation of AEC II, anti-ZONAB siRNA (siZONAB) was transfected to inhibit ZONAB expression. Indicators of AEC II transdifferentiation and proliferation were detected. The results indicated that ZONAB expression in siZONAB-transfected cells was significantly lower than that in the blank control group (transfected with nonspecific siRNA), and ZONAB expression in the nuclei was also significantly decreased $(\mathrm{P}<0.05 ;$ Fig. $4 \mathrm{~A})$. At the same time, the expression of the AEC I marker AQP5 was significantly increased and the expression of the AEC II marker SP-C was significantly decreased in the siZONAB-transfected group, suggesting increased transdifferentiation of the cells (Fig. 4B). PCNA and cyclin D1 expression levels were significantly decreased in the siZONAB-transfected group, indicating decreased cell proliferation (Fig. 4C). The double immunofluorescence staining results demonstrated that AQP5 expression was increased, SP-C expression was decreased and double-stained cells increased in the siZONAB-transfected group (Fig. 4D), suggesting increased transdifferentiation and decreased proliferation of AEC II in the siZONAB-transfected group. Cell viability was decreased in the siZONAB-transfected group compared with that in the control group (Fig. 4E).
ZONAB overexpression partially alleviates hyperoxia-induced abnormal transdifferentiation and proliferation of AEC II. To investigate whether ZONAB is involved in mediating the effects of hyperoxia on AEC II transdifferentiation and proliferation, the pCMV6-ZONAB plasmid or pCMV6 blank plasmid was transfected into primary AEC II, which were then exposed to hyperoxia or normoxia treatment to observe cell transdifferentiation and proliferation. ZONAB mRNA expression in the pCMV6-ZONAB-transfected and blank control groups was decreased after hyperoxia treatment. However, after hyperoxia treatment, the level of ZONAB expression in the pCMV6-ZONAB-transfected group was higher than that in the control group, but did not reach the concentration of that in the normoxic control group. AQP5 mRNA expression increased after hyperoxia treatment in each of the two groups, while the increase in the pCMV6-ZONAB-transfected group was significantly lower than that in the control group, suggesting that high ZONAB expression partially mitigated the excessive transdifferentiation caused by hyperoxia. SP-C and PCNA mRNA expression levels decreased in each of the two groups after hyperoxia treatment. Although the decrease in the pCMV6-ZONAB-transfected group was less significant 

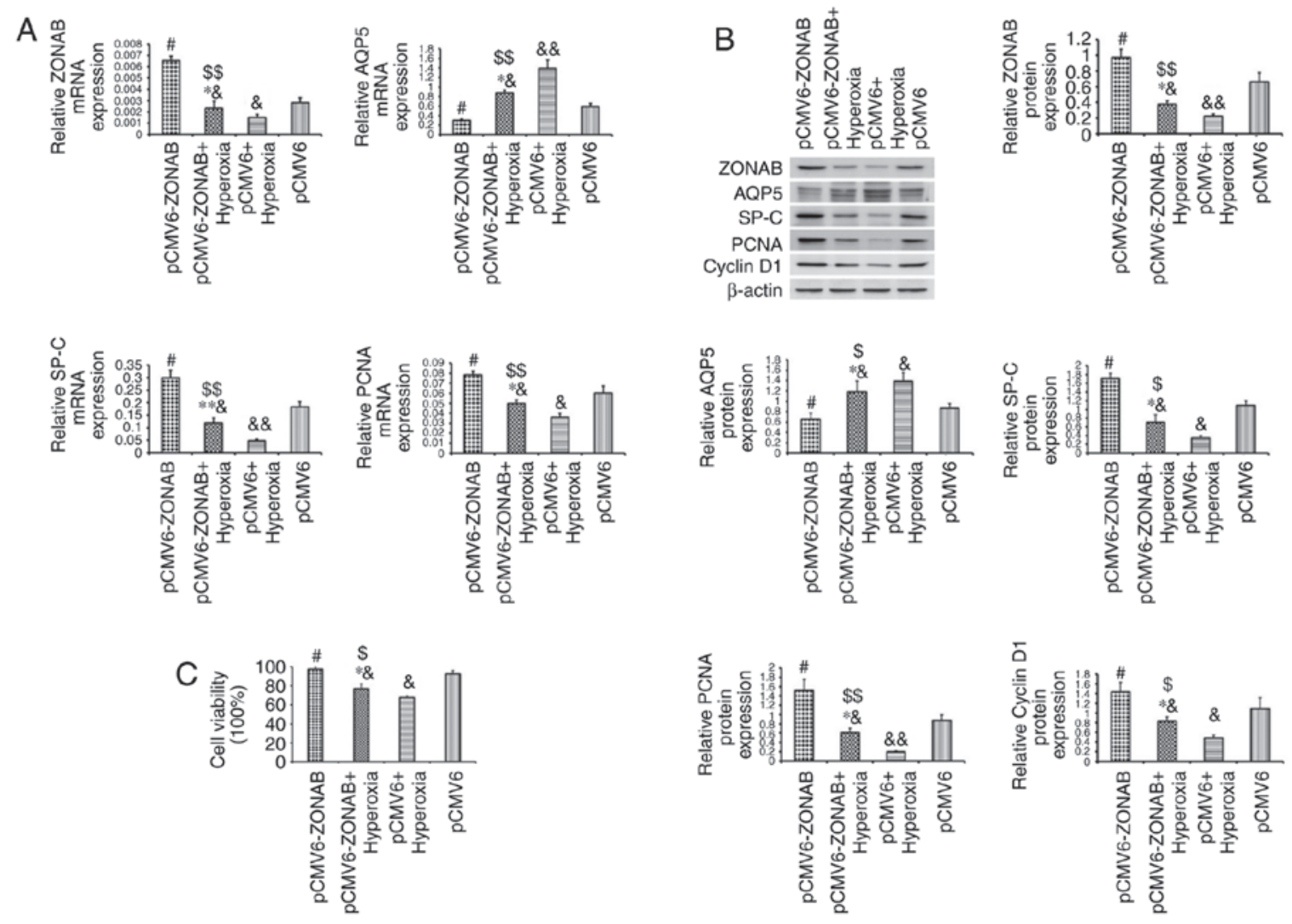

Figure 5. ZONAB overexpression partially alleviates hyperoxia-induced excessive transdifferentiation and decreased proliferation of AEC II. (A) mRNA expression levels of ZONAB, AQP5, SP-C and PCNA in primary AEC II of the pCMV6-ZONAB-transfected and blank control groups, with hyperoxia or normoxia exposure. (B) Protein expression of ZONAB, AQP5, SP-C, PCNA and cyclin D1 in each group. (C) Cell viability in each group. " $\mathrm{P}<0.05$ for comparison with the pCMV6 group, ${ }^{\$} \mathrm{P}<0.05,{ }^{\$ \$} \mathrm{P}<0.01$ for comparison with the pCMV6-ZONAB group, ${ }^{*} \mathrm{P}<0.05,{ }^{* * *} \mathrm{P}<0.01$ for comparison with the pCMV6+hyperoxia group, ${ }^{\&} \mathrm{P}<0.05$, \&\& $\mathrm{P}<0.01$ for comparison with the pCMV6 group. ZONAB, zonula occludens 1-associated nucleic acid binding protein; SP-C, surfactant protein C; AEC II, type II alveolar epithelial cells; PCNA, proliferating cell nuclear antigen; AQP, aquaporin; pCMV, cytomegalovirus plasmid.

than that in the pCMV6-transfected group under hyperoxia, it was still lower than that in the control group under normoxia conditions, suggesting that high ZONAB expression partially alleviated the hyperoxia-induced inhibition of proliferation (Fig. 5A). The changes in the protein expression of the above indicators were similar to the trends in the mRNA expression, and the changes in the protein expression of cyclin D1 were similar to those of PCNA (Fig. 5B). Hyperoxia caused significant cell death, and transfection of pCMV6-ZONAB partly alleviated the cell death (Fig. 5C).

\section{Discussion}

Previous studies on the pathogenesis of BPD mainly focused on AEC II injury, which is thought to be the pathological basis of BPD. Sureshbabu et al (25) reported that apoptosis of a large number of AEC II occurred in the BPD model. Roper et al (26) and O'Reilly et al (27) identified DNA damage in in vivo and in vitro models of BPD. Chen et al (28) demonstrated that AEC II produce a large number of reactive oxygen species under hyperoxia conditions, resulting in apoptosis and necrosis. However, the results of other studies differed from this. For instance, Crapo et al (29) revealed that the number of AEC II in BPD lung tissues was increased rather than decreased and Yee et al (8) reported that the number of AEC I increased in a hyperoxia-induced BPD model, suggesting that
AEC II injury is not the only mechanism of BPD injury in the lung epithelium. A previous study by our group demonstrated that transdifferentiation of AECs in the BPD model significantly increased at the tissue and cellular levels of AECs, accompanied by proliferative dysregulation (10). The transdifferentiation of AEC II to AEC I is an important mechanism in lung injury repair (30-32), but the elevated transdifferentiation level of AECs in the BPD model does not compensate for injury of the lung epithelium, in which AEC II as well as AEC I are structurally and functionally abnormal, resulting in destruction of the blood-gas barrier and opening of the pulmonary epithelial TJs (10). These changes are likely to be associated with excessive dysregulation of transdifferentiation and proliferation of AECs, which provides a novel pathway for further studies on the pathogenesis of BPD.

To further study the regulatory mechanism of the transdifferentiation and proliferation of AECs, the present study focused on the transcription factor ZONAB. A previous study by our group indicated decreased expression of ZO-1 in AECs in a model of hyperoxia-induced lung injury (33), which resulted in the opening of the TJs between AECs and destruction of the epithelial barrier. In addition to participating in the regulation of TJs, ZO-1 interacts with ZONAB to regulate cell differentiation and proliferation $(13,22,34)$. The role of ZONAB in the regulation of cell differentiation and proliferation has been studied in numerous diseases, including human hepatocellular 
carcinoma (35), uveal melanoma (36), proliferative vitreoretinopathy (37) and renal clear cell carcinoma (22). However, relatively few studies have assessed ZONAB expression in lung tissue and no study has investigated the effects of ZONAB on the onset and development of BPD. Therefore, the present study examined ZONAB expression in the lung tissues of rats with BPD and further confirmed that ZONAB participates in the regulation of the transdifferentiation and proliferation of AECs in vitro in order to explore the pathogenesis of BPD.

Evaluation of the western blot results of tissue samples indicated that ZONAB expression was greater in the in the lung tissues of the BPD model group as compared with that the control group. However, immunofluorescence double staining clearly demonstrated that ZONAB expression was decreased in AEC II of the model group. By isolating and purifying AEC II from neonatal rats and quantifying the protein and gene expression levels of ZONAB after subjecting the cells to hyperoxia in vitro, it was revealed that the ZONAB expression was reduced in the BPD model group, as compared with that the control group, indicating that ZONAB expression was unequally distributed in dozens of cells in the lung tissues of the hyperoxia-induced in vivo model of BPD. ZONAB expression may be increased in other cell types, including bronchial epithelial cells, as demonstrated by the immunofluorescence staining results. Other sources, such as fibroblasts, may have also contributed to the increased ZONAB expression in the in vivo model and are probably involved in the epithelial-mesenchymal transition $(10,38)$, although this speculation must be confirmed in future studies. In addition, the effect of hyperoxia on endogenous and exogenous ZONAB, as well as the mechanism of how hyperoxia causes a decrease in ZONAB in AEC II are questions requiring further exploration.

The regulatory effects of ZONAB on transdifferentiation and proliferation of AECs was further verified by manipulating ZONAB expression through gene transfection of primary AEC II. The results indicated that when ZONAB was overexpressed, the protein and mRNA expression levels of the AEC I marker AQP5 were downregulated, while those of the AEC II marker SP-C were upregulated, and the expression levels of PCNA and cyclin D1 were increased. The results of the immunofluorescence double staining also demonstrated that the transdifferentiation of AECs was decreased in the plasmid-transfected group, although a single channel and line scan for the intensity may be able to better demonstrate this, which should be included in a future study. The present results confirmed that overexpression of ZONAB inhibited the transdifferentiation and promoted the proliferation of AECs. In addition, the siZONAB transfection experiment confirmed that knockdown of ZONAB expression promoted the transdifferentiation and inhibited the proliferation of AECs. In the AECs, ZONAB inhibited transdifferentiation and promoted proliferation, which is consistent with the function of ZONAB in renal tubular epithelium (22), retina $(34,36,37)$, cornea (39), intestine (40), liver (35), mammary gland (18) and brain $(41,42)$, which is likely to be similar in lung tissue.

Given that ZONAB has a role in the regulation of AEC transdifferentiation and proliferation, and that excessive dysregulation of transdifferentiation and proliferation was observed in the BPD model (10), the present study investigated whether this dysregulation was mediated by ZONAB in the hyperoxia-induced BPD model. Since hyperoxia caused a decrease in ZONAB expression in AEC II, plasmids overexpressing ZONAB were transfected into AEC II, and the cells were then cultured under hyperoxic or normoxic conditions to further compare the cell differentiation and proliferation levels between the groups. The results indicated that ZONAB overexpression counteracted the effects of hyperoxia and partially attenuated the hyperoxia-induced dysregulation of AEC transdifferentiation and proliferation, but did not completely offset the effect of hyperoxia. Although the effects of ZONAB on transdifferentiation and proliferation of AECs are not hyperoxia-specific, it does take part in the hyperoxia-induced alveolar epithelial injury. These results indicated that the transdifferentiation and proliferation of AECs were partially dependent on ZONAB during the course of hyperoxia injury, although other factors may also be involved. Numerous factors participate in the regulation of AEC transdifferentiation and proliferation, and clinical studies on BPD patients and animal models reported abnormal expression of certain cytokines, including insulin-like growth factor I (43-45), transforming growth factor- $\beta 1$ (46-48), keratinocyte growth factor $(48,49)$ and bone morphogenetic protein $(46,50)$, which act to accelerate or inhibit AEC transdifferentiation $(45,51-53)$. Therefore, it is important to identify the factors involved in the mediation of AEC transdifferentiation and proliferation in the process of hyperoxia-induced BPD, and to investigate how these factors interact with one another and regulate cell transdifferentiation and proliferation.

In conclusion, the in vivo and in vitro experiments of the present study demonstrated that ZONAB expression was reduced in AEC II of the hyperoxia-induced BPD model. Transfection of ZONAB plasmids or siRNA confirmed that ZONAB inhibited the transdifferentiation and promoted the proliferation of AECs. Transfection of plasmids overexpressing ZONAB in AEC II ameliorated the hyperoxia-induced cell transdifferentiation and proliferation abnormalities in vitro, but did not completely offset the impact of hyperoxia, indicating that ZONAB is involved in mediating hyperoxia-induced BPD development of lung epithelial disorders. These results may provide a novel pathway to explore as a mechanism of pulmonary epithelial damage in $\mathrm{BPD}$, while the specific regulatory mechanisms of ZONAB on AEC transdifferentiation and proliferation remain to be elucidated.

\section{Acknowledgements}

This study was funded by the Natural Science Foundation of China (grant nos. 81601331 and 81471489).

\section{Competing interests}

The authors declare that they have no competing interests.

\section{References}

1. Islam JY, Keller RL, Aschner JL, Hartert TV and Moore PE: Understanding the short- and long-term respiratory outcomes of prematurity and bronchopulmonary dysplasia. Am J Respir Crit Care Med 192: 134-156, 2015.

2. Wang H, St Julien KR, Stevenson DK, Hoffmann TJ, Witte JS, Lazzeroni LC, Krasnow MA, Quaintance CC, Oehlert JW, Jelliffe-Pawlowski LL, et al: A genome-wide association study (GWAS) for bronchopulmonary dysplasia. Pediatrics 132: 290-297, 2013. 
3. Stoll BJ, Hansen NI, Bell EF, Shankaran S, Laptook AR, Walsh MC, Hale EC, Newman NS, Schibler K, Carlo WA, et al: Neonatal outcomes of extremely preterm infants from the NICHD Neonatal Research Network. Pediatrics 126: 443-456, 2010.

4. Bhandari A and McGrath-Morrow S: Long-term pulmonary outcomes of patients with bronchopulmonary dysplasia. Semin Perinatol 37: 132-137, 2013

5. Anderson PJ and Doyle LW: Neurodevelopmental outcome of bronchopulmonary dysplasia. Semin Perinatol 30: 227-232, 2006

6. Jobe AH and Bancalari E: Bronchopulmonary dysplasia. Am J Respir Crit Care Med 163: 1723-1729, 2001

7. Alvira CM: Aberrant pulmonary vascular growth and remodeling in bronchopulmonary dysplasia. Front Med (Lausanne) 3: 21, 2016.

8. Yee M, Buczynski BW and O'Reilly MA: Neonatal hyperoxia stimulates the expansion of alveolar epithelial type II cells. Am J Respir Cell Mol Biol 50: 757-766, 2014.

9. Thebaud B and Abman SH: Bronchopulmonary dysplasia: Where have all the vessels gone? Roles of angiogenic growth factors in chronic lung disease. Am J Respir Crit Care Med 175: 978-985, 2007.

10. Hou A, Fu J, Yang H, Zhu Y, Pan Y, Xu S and Xue X: Hyperoxia stimulates the transdifferentiation of type II alveolar epithelia cells in newborn rats. Am J Physiol Lung Cell Mol Physiol 308: L861-L872, 2015.

11. Yang H, Fu J, Xue X, Yao L, Qiao L, Hou A, Jin L and Xing Y: Epithelial-mesenchymal transitions in bronchopulmonary dysplasia of newborn rats. Pediatr Pulmonol 49: 1112-1123, 2014

12. Balda MS and Matter K: The tight junction protein ZO-1 and an interacting transcription factor regulate ErbB-2 expression. EMBO J 19: 2024-2033, 2000

13. Balda MS, Garrett MD and Matter K: The ZO-1-associated Y-box factor ZONAB regulates epithelial cell proliferation and cell density. J Cell Biol 160: 423-432, 2003.

14. Matter K and Balda MS: Epithelial tight junctions, gene expression and nucleo-junctional interplay. J Cell Sci 120 : 1505-1511, 2007.

15. Balda MS and Matter K: Tight junctions and the regulation of gene expression. Biochim Biophys Acta 1788: 761-767, 2009

16. Sherr CJ, Beach D and Shapiro GI: Targeting CDK4 and CDK6: From discovery to therapy. Cancer Discov 6: 353-367, 2016.

17. Xing Y, Fu J, Yang H, Yao L, Qiao L, Du Y and Xue X: MicroRNA expression profiles and target prediction in neonatal Wistar rat lungs during the development of bronchopulmonary dysplasia. Int J Mol Med 36: 1253-1263, 2015.

18. Sourisseau T, Georgiadis A, Tsapara A, Ali RR, Pestell R, Matter K and Balda MS: Regulation of PCNA and cyclin D1 expression and epithelial morphogenesis by the ZO-1-regulated transcription factor ZONAB/DbpA. Mol Cell Biol 26: 2387-2398, 2006.

19. Darzynkiewicz Z, Zhao H, Zhang S, Lee MY, Lee EY and Zhang Z: Initiation and termination of DNA replication during S phase in relation to cyclins D1,E and A, p21WAF1, Cdt1 and the p12 subunit of DNA polymerase delta revealed in individual cells by cytometry. Oncotarget 6: 11735-11750, 2015.

20. Frankel P, Aronheim A, Kavanagh E, Balda MS, Matter K, Bunney TD and Marshall CJ: RalA interacts with ZONAB in a cell density-dependent manner and regulates its transcriptional activity. EMBO J 24: 54-62, 2005.

21. Nielsen R, Christensen EI and Birn H: Megalin and cubilin in proximal tubule protein reabsorption: From experimental models to human disease. Kidney Int 89: 58-67, 2016.

22. Lima WR, Parreira KS, Devuyst O, Caplanusi A, N'kuli F, Marien B, Van Der Smissen P, Alves PM, Verroust P, Christensen EI, et al: ZONAB promotes proliferation and represses differentiation of proximal tubule epithelial cells. J Am Soc Nephrol 21: 478-488, 2010.

23. Livak KJ and Schmittgen TD: Analysis of relative gene expression data using real-time quantitative PCR and the 2(-Delta Delta C(T)) method. Methods 25: 402-408, 2001.

24. Mukherjee N, Cardenas E, Bedolla R and Ghosh R: SETD6 regulates NF-kB signaling in urothelial cell survival: Implications for bladder cancer. Oncotarget 8: 15114-15125, 2017.

25. Sureshbabu A, Syed M, Das P, Janér C, Pryhuber G, Rahman A, Andersson S, Homer RJ and Bhandari V: Inhibition of regulatory-associated protein of mechanistic target of rapamycin prevents hyperoxia-induced lung injury by enhancing autophagy and reducing apoptosis in neonatal mice. Am J Respir Cell Mol Biol 55: 722-735, 2016.
26. Roper JM, Mazzatti DJ, Watkins RH, Maniscalco WM, Keng PC and O'Reilly MA: In vivo exposure to hyperoxia induces DNA damage in a population of alveolar type II epithelial cells. Am J Physiol Lung Cell Mol Physiol 286: L1045-L1054, 2004.

27. O'Reilly MA, Staversky RJ, Finkelstein JN and Keng PC: Activation of the $\mathrm{G} 2$ cell cycle checkpoint enhances survival of epithelial cells exposed to hyperoxia. Am J Physiol Lung Cell Mol Physiol 284: L368-L375, 2003.

28. Chen Y, Chang L, Li W, Rong Z, Liu W, Shan R and Pan R: Thioredoxin protects fetal type II epithelial cells from hyperoxia-induced injury. Pediatr Pulmonol 45: 1192-1200, 2010.

29. Crapo JD, Barry BE, Foscue HA and Shelburne J: Structural and biochemical changes in rat lungs occurring during exposures to lethal and adaptive doses of oxygen. Am Rev Respir Dis 122: 123-143, 1980.

30. Barkauskas CE, Cronce MJ, Rackley CR, Bowie EJ, Keene DR, Stripp BR, Randell SH, Noble PW and Hogan BL: Type 2 alveolar cells are stem cells in adult lung. J Clin Invest 123: 3025-3036, 2013.

31. Lu HY, Shao GB, Li WB and Wang H: Effects of hyperoxia on transdifferentiation of primary cultured typeII alveolar epithelial cells from premature rats. In Vitro Cell Dev Biol Anim 47: 64-72, 2011.

32. Liebler JM, Marconett CN, Juul N, Wang H, Liu Y, Flodby P, Laird-Offringa IA, Minoo P and Zhou B: Combinations of differentiation markers distinguish subpopulations of alveolar epithelial cells in adult lung. Am J Physiol Lung Cell Mol Physiol 310: L114-L120, 2016.

33. You K, Xu X, Fu J, Xu S, Yue X, Yu Z and Xue X: Hyperoxia disrupts pulmonary epithelial barrier in newborn rats via the deterioration of occludin and ZO-1. Respir Res 13: 36, 2012.

34. Georgiadis A, Tschernutter M, Bainbridge JW, Balaggan KS, Mowat F, West EL, Munro PM, Thrasher AJ, Matter K, Balda MS and Ali RR: The tight junction associated signalling proteins ZO-1 and ZONAB regulate retinal pigment epithelium homeostasis in mice. PLoS One 5: e15730, 2010.

35. Arakawa Y, Kajino K, Kano S, Tobita H, Hayashi J, Yasen M, Moriyama M, Arakawa Y and Hino O: Transcription of dbpA, a Y box binding protein, is positively regulated by E2F1: Implications in hepatocarcinogenesis. Biochem Biophys Res Commun 322: 297-302, 2004.

36. Jayagopal A, Yang JL, Haselton FR and Chang MS: Tight junction-associated signaling pathways modulate cell proliferation in uveal melanoma. Invest Ophthalmol Vis Sci 52: 588-593, 2011.

37. Tamiya S and Kaplan HJ: Role of epithelial-mesenchymal transition in proliferative vitreoretinopathy. Exp Eye Res 142: 26-31, 2016.

38. Zhu Y, Fu J, Yang H, Pan Y, Yao L and Xue X: Hyperoxia-induced methylation decreases RUNX3 in a newborn rat model of bronchopulmonary dysplasia. Respir Res 16: 75, 2015.

39. Russ PK, Pino CJ, Williams CS, Bader DM, Haselton FR and Chang MS: Bves modulates tight junction associated signaling. PLoS One 6: e14563, 2011.

40. Nie M, Balda MS and Matter K: Stress- and Rho-activated ZO-1-associated nucleic acid binding protein binding to p21 mRNA mediates stabilization, translation, and cell survival. Proc Natl Acad Sci USA 109: 10897-10902, 2012.

41. Penes MC, Li X and Nagy JI: Expression of zonula occludens-1 (ZO-1) and the transcription factor $\mathrm{ZO}-1$-associated nucleic acid-binding protein (ZONAB)-MsY3 in glial cells and colocalization at oligodendrocyte and astrocyte gap junctions in mouse brain. Eur J Neurosci 22: 404-418, 2005.

42. Liu LB, Liu XB, Ma J, Liu YH, Li ZQ, Ma T, Zhao XH, Xi Z and Xue YX: Bradykinin increased the permeability of BTB via NOS/NO/ZONAB-mediating down-regulation of claudin-5 and occludin. Biochem Biophys Res Commun 464: 118-125, 2015.

43. Löfqvist C, Hellgren G, Niklasson A, Engström E, Ley D and Hansen-Pupp I; WINROP Consortium: Low postnatal serum IGF-I levels are associated with bronchopulmonary dysplasia (BPD). Acta Paediatr 101: 1211-1216, 2012.

44. Hellström A, Ley D, Hansen-Pupp I, Hallberg B, Ramenghi LA, Löfqvist C, Smith LE and Hård AL: Role of insulinlike growth factor 1 in fetal development and in the early postnatal life of premature infants. Am J Perinatol 33: 1067-1071, 2016.

45. Ghosh MC, Gorantla V, Makena PS, Luellen C, Sinclair SE, Schwingshackl A and Waters CM: Insulin-like growth factor-I stimulates differentiation of ATII cells to ATI-like cells through activation of Wnt5a. Am J Physiol Lung Cell Mol Physiol 305: L222-L228, 2013. 
46. Alejandre-Alcázar MA, Kwapiszewska G, Reiss I, Amarie OV, Marsh LM, Sevilla-Pérez J, Wygrecka M, Eul B, Köbrich S, Hesse M, et al: Hyperoxia modulates TGF-beta/BMP signaling in a mouse model of bronchopulmonary dysplasia. Am J Physiol Lung Cell Mol Physiol 292: L537-L549, 2007.

47. Luan Y, Zhang L, Chao S, Liu X, Li K, Wang Y and Zhang Z: Mesenchymal stem cells in combination with erythropoietin repair hyperoxia-induced alveoli dysplasia injury in neonatal mice via inhibition of TGF- $\beta 1$ signaling. Oncotarget 7 : 47082-47094, 2016

48. Been JV, Debeer A, van Iwaarden JF, Kloosterboer N, Passos VL, Naulaers G and Zimmermann LJ: Early alterations of growth factor patterns in bronchoalveolar lavage fluid from preterm infants developing bronchopulmonary dysplasia. Pediatr Res 67: 83-89, 2010.

49. Franco-Montoya ML, Bourbon JR, Durrmeyer X, Lorotte S, Jarreau PH and Delacourt C: Pulmonary effects of keratinocyte growth factor in newborn rats exposed to hyperoxia. Am J Physiol Lung Cell Mol Physiol 297: L965-L976, 2009.
50. Hines EA and Sun X: Tissue crosstalk in lung development. J Cell Biochem 115: 1469-1477, 2014.

51. Bhaskaran M, Kolliputi N, Wang Y, Gou D, Chintagari NR and Liu L: Trans-differentiation of alveolar epithelial type II cells to type I cells involves autocrine signaling by transforming growth factor beta 1 through the Smad pathway. J Biol Chem 282: 3968-3976, 2007.

52. Zhao L, Yee M and O'Reilly MA: Transdifferentiation of alveolar epithelial type II to type I cells is controlled by opposing TGF- $\beta$ and BMP signaling. Am J Physiol Lung Cell Mol Physiol 305: L409-L418, 2013.

53. Qiao R, Yan W, Clavijo C, Mehrian-Shai R, Zhong Q, Kim KJ, Ann D, Crandall ED and Borok Z: Effects of KGF on alveolar epithelial cell transdifferentiation are mediated by JNK signaling. Am J Respir Cell Mol Biol 38: 239-246, 2008. 